\title{
Antiretroviral Therapy Switch Rates and Switching Patterns In 16,069 People Living with HIV: A Nationwide, Population-Based Study in Japan
}

Toshio Naito ( $\nabla$ naito@juntendo.ac.jp ) Juntendo University Faculty of Medicine Hirotake Mori Juntendo University Faculty of Medicine Kazutoshi Fujibayashi Juntendo University Faculty of Medicine Shinichi Fukushima Juntendo University Faculty of Medicine Mayumi Yuda Juntendo University Faculty of Medicine Nobuyuki Fukui Juntendo University Faculty of Medicine Shotaro Tsukamoto Juntendo University Faculty of Medicine Mai Suzuki Juntendo University Faculty of Medicine Keiko Goto-Hirano Juntendo University Faculty of Medicine Ryohei Kuwatsuru Juntendo University Faculty of Medicine

\section{Research Article}

Keywords: Antiretroviral therapy, Switch rate, Time-to-switch, Integrase strand transfer inhibitor, National Database of Health Insurance Claims and Specific Health Checkups of Japan, Claims database study, Asia, Japan

Posted Date: July 10th, 2021

DOl: https://doi.org/10.21203/rs.3.rs-691735/v1 
License: (c) (i) This work is licensed under a Creative Commons Attribution 4.0 International License. Read Full License 


\section{Abstract}

To report the status of switch rates and time-to-switch of antiretroviral therapy (ART) regimens by evaluating anchor drug classes and common switching patterns in Japanese people living with human immunodeficiency virus (HIV, PLWH). This cross-sectional cohort study extracted data of 28,089 PLWH from the National Database of Health Insurance Claims and Specific Health Checkups of Japan (NDB), which contains data representing the entire population of Japan. PLWH with first prescription records of ART administered between January 2011 and March 2019 were identified $(n=16,069)$. The median timeto-switch and switch rates of anchor drug classes were estimated by Kaplan-Meier analysis. BrookmeyerCrowley and Greenwood methods were used to estimate $95 \%$ confidence intervals for switch rates and median days, respectively. Switch rates were compared between anchor drug classes by year using logrank tests. A total of 3,108 (19.3\%) PLWH switched anchor drug classes from first to second regimens. Switch rates increased continuously over eight years for non-nucleoside reverse transcriptase inhibitors (NNRTIs) (14.9\%-65.5\%) and protease inhibitors (PIs) (13.2\%-67.7\%), with median time-to-switch of 1,826 and 1,583 days, respectively. Integrase strand transfer inhibitors (INSTIs) maintained a low switch rate $(3.0 \%-7 \cdot 6 \%)$, precluding median-days calculation. The majority of patients treated initially with NNRTIs and PIs switched to INSTIs regardless of switching times (< 1 year: $67.3 \%$ and $85.9 \%$, respectively; $\geq 1$ year: $95.5 \%$ and $93.6 \%$, respectively). The foremost switching strategies for first-to-second ART regimens are from NNRTI or PI to INSTI regimens that maintain low switch rates long term. INSTI HIV agents may be the most durable anchor drug class for PLWH receiving ART.

\section{Introduction}

Antiretroviral drugs are being used globally to treat people living with human immunodeficiency virus (HIV, PLWH). International and national guidelines stipulate that durable, straightforward antiretroviral therapy (ART) regimens are the main focus of lifelong chronic HIV treatment. ${ }^{1-3}$ Administration of ART regimens supports immune system function, reduces complications, and improves quality of life, ${ }^{4}$ decreasing morbidity and mortality. Increased survival rates among PLWH are attributed to successful ART. ${ }^{5-7}$ Life expectancy for PLWH now approximates that of HIV-negative individuals. ${ }^{8,9}$

Nevertheless, PLWH frequently switch ART regimens during chronic HIV treatment. Changes may occur because patients or clinicians think a new medication may produce better results or patients' may become dissatisfied with their current regimen. Drug toxicity, unsatisfactory levels of viral suppression, drug-related adverse events (AEs), or just simplification of a regimen may also prompt a switch. ${ }^{10-12}$ However, no consensus exists regarding switching strategies, and though changes occur frequently, switching strategies remain to be clearly defined.

The absence of a standard switching strategy makes it imperative to fully understand the circumstances most often leading to ART regimen switches and the anchor drug classes involved. Anchor drug classes that tend to be administered for longer durations also need to be identified. 
Five classes of antiretroviral medications are used in Japan: nucleoside reverse transcriptase inhibitors (NRTIs), nucleoside reverse transcriptase inhibitors (NNRTIs), protease inhibitors (PIs), integrase strand transfer inhibitors (INSTIs), and entry inhibitors (Els). ${ }^{3}$ The most recent guidelines for initiating an ART regimen recommend combination regimens (CART) consisting of two NRTIs as backbone therapy, with a third "anchor" drug from another class, most often NNRTIs, PIs, or INSTIs. ${ }^{1-3}$ Clinicians' selection of an anchor drug is central to the treatment strategy because backbone choices are comparatively limited.

Our previous study was a preliminary assessment of switch rates and time-to-switch of ART regimens using a hospital claims database with a distinctly smaller dataset of eligible HIV-positive patients than that in the larger nationwide dataset used for the present study. ${ }^{13}$ We hypothesized that expanding the data source would not only confirm our preliminary results, but also update our knowledge of current switching rates and patterns observed nationwide. Therefore, we used the nationwide database, the National Database of Health Insurance Claims and Specific Health Checkups of Japan (NDB), which contains data representing the entire population in Japan, to confirm and update our preliminary findings and identify the most effective ART approaches for long-term treatment of PLWH in Japan.

\section{Methods}

\section{Study design and data source}

This observational, retrospective cohort study extracted patient data from the Japanese National Database (NDB) for HIV-positive patients who received treatment between April 2009 and March 2019. ${ }^{14}$ The NDB is the largest nationwide cross-sectional database in Japan, and it contains comprehensive health insurance claims records from the National Health Insurance system of Japan for direct primary care delivered as inpatient care. The NDB has been used to supported clinical studies. ${ }^{15,16}$ The NDB includes data for diagnoses, age, sex, dates of outpatient services, dates of admission and discharge, procedures undertaken, prescribed drugs, etc. Patients treated from April 2009 to March 2019 with at least one diagnosis and any treatment processed in the claims data were enrolled. All included diagnoses were categorized according to the "The International Classification of Disease, 10th Revision, Clinical Modification" (ICD-10) diagnostic system. Patients with a diagnostic code of HIV-2 infection were excluded.

All insurance claims data are deidentified by the Ministry of Health, Labour and Welfare, and the ministry's guidelines on information security were followed in the study. To ensure patient privacy, inspection by and permission from the Ministry for publication is required before submission of the draft manuscript.

\section{Study population}

HIV-positive patients in the database were identified by the presence of at least one record of the International Classification of Diseases 10th Revision (ICD-10) codes B20-24, including: HIV disease 
resulting in infectious and parasitic diseases (B20), malignant neoplasms (B21), other specified diseases (B22), other conditions (B23), and unspecified HIV disease (B24). To avoid including doubtful HIV-positive patients (i.e., poorly recorded or intentionally recorded for a claim), patients were required to have at least one prescription record of ART. ART was defined as a prescription for any of the following antiretroviral drugs: NRTIs, NNRTIs, PIs, INSTIs, or Els.

Data of patients meeting these criteria $(n=28,089)$ during the study period were extracted from the database. Patients who had received first prescriptions for an ART regimen between 2011 and 2019 $(n=16,069)$ were included in the present analysis.

\section{Outcomes}

Primary outcomes were switch rates and time-to-switch associated with individual ART regimens by anchor drug classes. Secondary outcomes were switch rates and time-to-switch associated with anchor drug class-based ART regimens by type of backbone drug, characteristics of patients who experienced an anchor drug switch in each anchor drug class-based ART regimen, and common switching patterns of anchor drug classes.

\section{Definitions}

Data extracted from the NDB included patients' demographic characteristics (age, sex), and clinical characteristics, including year of first ART record in the database, prescription records of anchor drugs and backbone drugs, comorbidities, hospitalization history, and AIDS-defining illnesses.

\section{Anchor drugs and backbone drugs}

The anchor drugs of the ART regimens were identified using receipt codes and were classified into three anchor drug classes according to the anti-HIV drug classification available in Japan: ${ }^{3}$ 1) NNRTIs, 2) PIs, or 3) INSTIs.

The backbone drugs of the ART regimens were identified using receipt codes and classified into four categories: 1) tenofovir disoproxil fumarate (TDF); 2) abacavir (ABC); 3 ) tenofovir alafenamide fumarate (TAF); and 4) others.

\section{ART regimen switch and time-to-switch}

An ART regimen switch involved only anchor drug classes and was defined on the basis of a switch in the specific anchor drug class used in the ART regimen. The time-to-switch of an ART regimen was defined as the period from the date of the first record of anchor drug class in the ART regimen (defined as the first regimen) recorded within patient data (index date) to the date of switching to another anchor drug class in the subsequent ART regimen (defined as the second regimen) during the study period. The date of an anchor drug class switch was defined as the date of prescription of the new anchor drug class after the termination of the preceding (first) ART regimen. A regimen was considered discontinued when no 
initiation of any new anchor drug class was identified after termination of the preceding (first) ART regimen. A change of anchor drug within the same anchor drug class was not considered a switch.

\section{AIDS-defining illnesses}

AIDS-defining illnesses were identified by the presence of any of the following records prior to index date: HIV non-tuberculous mycobacteria, HIV cytomegalovirus infection, HIV candidiasis, HIV Pneumocystis carinii pneumonia, HIV Kaposi's sarcoma, HIV Burkitt's lymphoma, HIV non-Hodgkin's lymphoma, HIV encephalopathy, HIV-associated dementia, slim disease, acquired immune deficiency syndrome, AIDS, neonatal HIV infection, and AIDS-related complex. The corresponding ICD-10 codes are listed in Supplementary Table 1.

\section{Comorbidities}

Comorbidities were identified if any ICD-10-coded chronic illnesses were present prior to the index date, including: HIV-related diseases, hypertension, dyslipidemia, hepatitis $\mathrm{B} / \mathrm{C}$ coinfection, diabetes mellitus, bone disorder, vascular disease, psychiatric disorders, kidney disease, malignancy, and syphilis. Corresponding ICD-10 codes are listed in Supplementary Table 1.

\section{History of hospitalization}

A history of hospitalization was identified if a record of hospitalization was present before the ART regimen was prescribed.

\section{Statistical analysis}

The proportions of anchor drug class-based and backbone-based drugs prescribed on the index date were obtained by year. Demographic and clinical characteristics of all patients on ART regimens were analyzed descriptively according to the anchor drug class prescribed on the index date. The median time-to-switch and switch rates according to anchor drug class prescribed on the index date and those stratified by the backbone drugs were estimated using Kaplan-Meier analysis. To estimate 95\% confidence interval (Cls), the Brookmeyer and Crowley method was used for the median number of days, and the Greenwood method was used for switch rates. Log-rank tests were used to compare switch rates between the respective drug classes in each year. The Bonferroni method was performed to adjust $p$-values on multiple comparison. Discontinuation or continuation of the regimen to the end of the study period was censored.

The demographic and clinical characteristics of patients who switched anchor drug classes in their ART regimens were analyzed descriptively according to the anchor drug class prescribed on the index date. Timings of $<1$ and $\geq 1$ year were analyzed descriptively for patients who switched anchor drug classes in their ART regimens according to the anchor drug class prescribed on the index date and the corresponding $95 \% \mathrm{Cl}$ using Wilson scores. 
Confounding factors for switching ART regimens and factors interacting with the anchor drug class were selected a priori based on previous studies. Time-to-switch was an objective variable and the anchor drug class, risk factors, and interaction term between anchor drug classes and each risk factor were included as explanatory variables in this model. The hazard ratio (HR) of each anchor drug class was calculated after adjusting for remaining variables and stratified by interaction factors to estimate the risk of switching anchor drug classes from the ART regimen prescribed on the index date.

All statistical analyses were performed in the R 4.0.3 environment (R Core Team, 2020). All remaining statistical tests were two-sided, and $p<0.05$ indicated significance.

\section{Results}

\section{Patient disposition}

A total of 28,089 patients in the database had a coded diagnostic record of HIV during the study period. Of these, 27,912 were prescribed anchor drugs. After excluding 866 patients for whom multiple anchor drug classes were prescribed on the index date and patients with prescription records of El as the first anchor drug, 27,046 patients had a single prescription record of an anchor drug class on the index date. Broad use of the INSTI class in the database started after 2010, two years after INSTIs were introduced in Japan in July 2008. Therefore, the main results reported in the present study are derived from patients who started an ART regimen between 2011 and $2019(n=16,069)$ (Supplementary Figure 1).

\section{Distribution of anchor drug class and backbone drugs in ART regimens by year}

Prescriptions for NNRTIs and PIs as anchor drug classes in overall ART regimens decreased after 2011 (NNRTIs: 18\%-1\% from 2011 to 2019; PIs: 52\%-4\% from 2011 to 2019) (Fig. 1a). Contrary to these trends, prescriptions for INSTIs increased rapidly since 2011 and accounted for the majority of anchor drug classes prescribed in 2019 (30\% in 2011 and 95\% in 2019). Among backbone drug types, changes were observed starting from 2016. TDF was the predominant backbone drug type between 2011 and $2016(80 \%-50 \%)$, followed by ABC (14\% in 2011 to $38 \%$ in 2016) (Fig. 1b). TAF was the predominant backbone drug type from 2017 to 2019 (55\% in 2017 to $70 \%$ in 2019).

Of 16,069 patients who started an ART regimen during 2011-2019, 7·5\% $(n=1,204)$ were on NNRTIs, $24.3 \%(n=3,901)$ on PIs, and $68 \cdot 2 \%(n=10,964)$ on INSTIs (Supplementary Table 2$)$. Patients on INSTIs had higher proportions of psychiatric disorders compared to patients on NNRTIs and PIs (Supplementary Table 2).

\section{Characteristics of patients who switched anchor drug classes}

Of all patients who started an ART regimen from 2011 to 2019, 19.3\% (3,108 patients) switched anchor drug classes. For each anchor drug class, $47 \cdot 1 \%$ switched from NNRTIs $(568 / 1,204), 52 \cdot 7 \%$ from PIs $(2,057 / 3,901)$, and $4 \cdot 4 \%$ from INSTIs $(483 / 10,964)$ (Table 1$)$. No significant differences were found in patients' characteristics between those treated with these three predominant anchor drug classes. AIDS- 
defining illnesses were present in $60.5 \%$ of patients treated initially with INSTIs, whereas the proportions were lower in those treated initially with NNRTIs or PIs (51.4\% and $50.9 \%$, respectively). The proportion of patients with vascular diseases was highest among patients treated initially with INSTIs (8.3\%) compared to those treated initially with NNRTIs $(4 \cdot 4 \%)$ or PIs (3.5\%). The proportion of patients with psychiatric disorders was highest among patients treated initially with INSTIs (27.3\%), followed by those with PIs (16.4\%) and NNRTIs (14.6\%) (Table 1$)$.

\section{Switching patterns of anchor drug classes}

Of the 3,108 patients who switched anchor drug classes from their first to second ART regimens (Fig. 1c), most patients treated initially with NNRTIs and PIs switched to INSTIs $(67 \cdot 3 \%$ [95\% Cl: $63 \cdot 9 \%-70 \cdot 4 \%]$ and $85 \cdot 9 \%[84 \cdot 4 \%-87 \cdot 3 \%]$, respectively) $<1$ year after starting the first regimen; $65 \cdot 6 \%$ [95\% Cl: $63 \cdot 1 \%-68 \cdot 0 \%$ ) of patients treated initially with INSTIs switched to PIs in the second regimen. Of the patients who switched their anchor drug class $\geq 1$ year after starting their first regimen, most of those treated initially with NNRTIs and PIs switched to INSTIs (95.5\%, [95\% Cl: 94.4\%-96.3\%] and 93.6\% [95\% Cl: $93 \cdot 0 \%-$ $94 \cdot 1 \%]$, respectively), whereas of those treated initially with INSTIs, $55 \cdot 4 \%$ [95\% Cl: $52 \cdot 0 \%-58 \cdot 8 \%$ ] switched to Pls.

\section{Switching of anchor drug classes in the ART regimens}

The switch rates for both NNRTIs and Pls increased constantly over eight years (from $14.9 \%$ to $65.5 \%$ and $13 \cdot 2 \%$ to $67 \cdot 7 \%$, respectively), whereas patients taking INSTIs maintained a low switch rate (from $3 \cdot 0 \%$ to 7.6\%) (Fig. 2). NNRTIs and PIs had median time-to-switch of 1,826 and 1,583 days, respectively, but INSTIs had a low switch rate of $7 \cdot 6 \%$ at eight years, so time-to-switch could not be obtained. Log-rank tests showed significant differences in switch rates of any pairs of anchor drug classes at years one through eight (all $p<0.05)$.

\section{Switching of anchor drug class-based backbone drugs in the ART regimens}

In patients receiving NNRT Is, the switch rates at one year varied between backbone drugs, with the lowest rate in $A B C$ backbone drugs (14.0\%). In patients receiving Pls, the lowest rate was for TDF backbone drugs (10.7\%) (Fig. 3). In the PI group, significant differences were found in the switching rates between TDF and ABC and between TDF and other backbone drugs (all $p<0 \cdot 05$ ). In the INSTI group, equally low switch rates were observed at one year for TAF (2.1\%), ABC (2.8\%), and TDF (3.2\%). The switch rate for TAF was slightly lower than for TDF and ABC; the highest switch rate was using other backbone drugs $(9 \cdot 4 \%)$.

\section{Assessment of potential confounding factors associated with regimen switch}

AIDS-defining illness, backbone type, and anchor drug class, and interaction terms between anchor drug class and AIDS-defining illness and between anchor drug class and type of backbone were selected for inclusion in the Cox regression analysis model. Subsequently, HRs were calculated for anchor drug class stratified by each interaction term with anchor drug class (AIDS-defining illness and backbone types). The 
HRs were consistently higher in regimens with PIs and NNRTIs compared with those with INSTIS, regardless of the presence of AIDS-defining illness (HRs 7.70-9.06 for presence of AIDS, 13.07-13.14 for non-AIDS) or backbone type (HRs 11.39-11.78 for TAF, 10.84-11.05 for TDF, and 9.33-14.25 for ABC) (Fig. 4).

\section{Discussion}

The present study is the first to use the cross-sectional, nationwide Japanese NDB to analyze treatment data of HIV-positive Japanese persons. Analyses of switch rates, time-to-switch, and switching patterns of anchor drug classes of ART regimens showed that the most common switching pattern of anchor drug class was from NNRTIs or PIs to INSTIs. Switch rates for NNRTIs and PIs increased continuously over eight years, whereas initial INSTI regimens maintained low switch rates, validating the results of our preliminary study. ${ }^{13}$

Among PLWH enrolled in the present study, INSTI was the anchor drug class prescribed most frequently, and TAF agents (about two-thirds of backbone prescriptions) were the predominant initial ART regimen of the latest 3 years. The 2017 HIV treatment guidelines published in Japan ${ }^{17}$ reinforced these trends for initial ART regimens, showing that prescriptions for INSTI agents (e.g., raltegravir [RAL] and dolutegravir [DTG]) as initial anchor drug classes increased between 2012 and 2016; TDF was prescribed most frequently as a backbone drug followed by $A B C$ in the same timeframe. There were some concerns regarding switching of backbone drugs in the literature; PLWH who switched from a TDF-based to a TAFbased cART regimen showed increased low-density lipoprotein (LDL) values exceeding their cardiovascular risk targets. ${ }^{18,19}$ In the present study, equally low switch rates were observed at one year for TAF, ABC, and TDF. However, the true impact of TAF on lipid profiles or cardiovascular risk was not evaluated and requires further study.

Over the eight-year study period, the switch rates of anchor drug classes NNRTIs and PIs increased steadily. Switching anchor drug class also increased 20\% over three years in Europe and the United States in treatment-naïve PLWH treated initially with both NNRTIs and PIs. ${ }^{12}$ The majority of patients in the present study who were treated initially with NNRTIs and PIs switched to INSTIs regardless of switching times and/or backbone drugs, with low switch rates thereafter. These findings and similar findings reported by other investigators ${ }^{20-22}$ suggest that INSTIs may be the most durable anchor drug class for PLWH on ART regimens, regardless of backbone drugs in the first ART regimen. Analysis of a large dataset of HIV-positive patients confirmed that initial INSTI-based regimens combined with TDF, TAF, or $A B C$ were all potent and well tolerated without significant virological failure; only a small percentage of patients (12\%) discontinued INSTI regimens, and DTG showed the lowest risk of virological failure. ${ }^{23}$ The durability and efficacy of DTG were also reported in patients who switched to INSTI-based regimens, and subsequent switches were less likely than with RAL. ${ }^{22}$ Switching regimens appears to be more stable in virologically suppressed HIV-1-infected patients who receive INSTI-based regimens initially. ${ }^{21,24}$ 
Other possible explanations suggested for increased switching include the expansion of HIV/AIDS treatment programs in middle-income or resource-limited areas. ${ }^{25}$ In addition, HIV-infected patients are more likely to be younger, less educated, and to have detectable HIV-1 DNA when switching to a secondline cART regimen, which may predispose to worse outcomes. Comparison of outcomes of second-line CART regimens between 1996-1998 and 2008-2010 reported that failure rates decreased as time progressed and were independent of the CART regimen; risk of virologic failure of second-line CART was also lower in patients who had undetectable HIV-1 DNA at the time of switching. ${ }^{26}$ Increases in ART drug resistance may also explain multiple switches in treatment. ${ }^{27}$

Of comorbidities, the prevalence of AIDS-defining illnesses was the highest in those treated initially with INSTIs, whereas dyslipidemia and diabetes mellitus prevalence was higher among patients receiving NNRTIs. Multivariate analysis, after adjusting for confounders, showed that the risk of switching anchor drug classes was lower in patients prescribed INSTIs, regardless of the presence of AIDS-defining illness or type of backbone prescribed, supporting long-term continuation of INSTIs prescribed for the first ART regimen.

\section{Limitations}

The present study has several limitations, including its cross-sectional design and retrospective analysis, which both limit inferences of causality. The study population was confined to Japan, and thus the results cannot be generalized to other populations. Furthermore, patients with more comorbid chronic illnesses or more complications of HIV infection may have been hospitalized in HIV-specialized institutions or institutions offering advanced medical care, which may limit generalizing results to all PLWH throughout Japan. Data were from an administrative database and certain clinical data from individual patients (e.g., adverse events, treatment failure, poor adherence) may be unavailable to accurately determine ART regimen changes or drug selection. A prospective long-term study is needed to confirm the durability of INSTIs as initial ART regimen drugs.

\section{Conclusions}

This is the first report of switch rates and time-to-switch of ART regimens using a NDB, which not only contains information on the largest number of HIV-positive patients in Japan, but also is representative of the entire population in Japan. The foremost switching strategies for first-to-second ART regimens were from NNRTIs or PIs to INSTIs. Incorporating INSTIs as the anchor drugs in initial ART regimens maintains low switch rates for long durations, suggesting that INSTIs may be the most durable anchor drug class for PLWH on ART regimens, regardless of AIDS-defining illnesses or backbone drug types prescribed.

\section{Declarations}

\section{Sources of Funding}


This work was supported by the Ministry of Health, Labour and Welfare (MHLW) Research on HIV/AIDS Program Grant Number JPMH20HB2001. However, this research has no conflict of interest.

\section{Authors' contributions}

$\mathrm{TN}, \mathrm{KF}$, and SF contributed to the study design. MY, NF, and ST contributed to the analysis and interpretation of the data. TN and SF had verified the underlying data. TN, HM, MS, KGH, and RK critically revised the draft manuscript and approved the final version of the manuscript for publication.

\section{Declaration of interests}

There are no conflicts of interest.

Funding: This work was supported by Ministry of Health, Labor and Welfare Research on HIV/AIDS Program Grant Number JPMH20HB2001.

\section{References}

1. European, A. I. D. S. C. \& Society European Guidelines for Clinical Management and Treatment of HIV1-Infected Adults in Europe, Version 9.1. Available at:

http://www.eacsociety.org/files/2018_guidelines-9.1-english.pdf.

2. DHHS Panel on Antiretroviral Guidelines for Adults and Adolescents. Guidelines for the use of antiretroviral agents in HIV-1-infected adults and adolescents. Department of Health and Human Services. Updated on October 25, 2018. Available at: https://aidsinfo.nih.gov/contentfiles/lvguidelines/adultandadolescentgl.pdf

3. The Guidelines for the Treatment of HIV Infection, March 2019 version. Japanese Ministry of Health, Labour and Welfare. 1-156. Available at: https://www.haart-support.jp/guideline.htm

4. Oguntibeju, O. O. Quality of life of people living with HIV and AIDS and antiretroviral therapy. HIV AIDS (Auckl), 4, 117-124 https://doi.org/https:10.2147/HIV.S32321 (2012).

5. Antiretroviral Therapy Cohort Collaboration. Life expectancy of individuals on combination antiretroviral therapy in high-income countries: a collaborative analysis of 14 cohort studies., $\mathbf{3 7 2 ,}$ 293-299 (2008).

6. Costagliola, D. Demographics of HIV and aging. Curr Opin HIV AIDS, 9, 294-301 (2014).

7. Samji, H. et al. North American AIDS Cohort Collaboration on Research and Design (NA-ACCORD) of leDEA. Closing the gap: increases in life expectancy among treated HIV-positive individuals in the United States and Canada. PLoS One, 8, e81355 (2013).

8. Nakagawa, F., May, M. \& Phillips, A. Life expectancy living with HIV: recent estimates and future implications. Curr Opin Infect Dis, 26, 17-25 (2013).

9. van Sighem, A. I., Gras, L. A., Reiss, P., Brinkman, K. \& de Wolf, F. ATHENA national observational cohort study. Life expectancy of recently diagnosed asymptomatic HIV-infected patients approaches 
that of uninfected individuals. AIDS, 24, 1527-1535 (2010).

10. Elzi, L. et al. Swiss HIV Cohort Study. Treatment modification in human immunodeficiency virusinfected individuals starting combination antiretroviral therapy between 2005 and 2008. Arch Intern Med, 170, 57-65 (2010).

11. Prosperi, M. C. et al. Predictors of first-line antiretroviral therapy discontinuation due to drug-related adverse events in HIV-infected patients: a retrospective cohort study. BMC Infect Dis, 12, 296 (2012).

12. Abgrall, S. et al. Antiretroviral Therapy Cohort Collaboration (ART-CC). Durability of first ART regimen and risk factors for modification, interruption or death in HIV-positive patients starting ART in Europe and North America 2002-2009. AIDS, 27, 803-813 (2013).

13. Ruzicka, D. J., Kuroishi, N., Oshima, N., Sakuma, R. \& Naito, T. Switch rates, time-to-switch, and switch patterns of antiretroviral therapy in people living with human immunodeficiency virus in Japan, in a hospital-claim database. BMC Infect Dis, 19, 505 (2019).

14. Ministry of Health, Labour and Welfare. NDB Open Data [in Japanese]. Available at https://www.mhlw.go.jp/stf/seisakunitsuite/bunya/0000177182.html Accessed December 2019.

15. Nojiri, S. et al. Comorbidity status in hospitalized elderly in Japan: Analysis from National Database of Health Insurance Claims and Specific Health Checkups. Sci Rep, 9, 20237 (2019).

16. Hirose, N., Ishimaru, M., Morita, K. \& Yasunaga, H. A review of studies using the Japanese National Database of Health Insurance Claims and Specific Health Checkups. Ann Clin Epidemiol, 2, 13-26 https://doi.org/https:10.37737/ace.2.1_13 (2020).

17. The Guidelines for the Treatment of HIV Infection, March 2017 version.The Japanese Ministry of Health, Labour and Welfare.1-152.

18. Gazzola, L. et al. Dyslipidaemia after switch to tenofovir alafenamide (TAF)-based cART regimens in a cohort of HIV-positive patients: what clinical relevance? HIV Med, 22, 140-145 https://doi.org/https:10.1111/hiv.12984 (2021).

19. Byonanebye, D. M. \& RESPOND Study Group. Incidence of dyslipidemia in people with HIV who are treated with integrase inhibitors versus other antiretroviral agents. AIDS, 35, 869-882 https://doi.org/https:10.1097/QAD.0000000000002811 (2021).

20. Varriano, B. et al. Assessment of antiretroviral third agent virologic durability after initiation of first antiretroviral regimen. Int J STD AIDS, 30, 680-688 https://doi.org/https:10.1177/0956462418815292 (2019).

21. Raffi, F., Esser, S., Nunnari, G., Pérez-Valero, I. \& Waters, L. Switching regimens in virologically suppressed HIV-1-infected patients: evidence base and rationale for integrase strand transfer inhibitor (INSTI)-containing regimens. HIV Med, Suppl 5, 3-16 https://doi.org/https:10.1111/hiv.12440 (2016).

22. Giacomelli, A. et al. Durability of INI-containing regimens after switching from PI-containing regimens: a single-centre cohort of drug-experienced HIV-infected subjects. Drug Des Devel Ther, 13, 2271-2282 https://doi.org/https:10.2147/DDDT.S204415 (2019). 
23. D'Monforte, A. et al. Durability of first-line regimens including integrase strand transfer inhibitors (INSTIs): data from a real-life setting. J Antimicrob Chemother, 74, 1363-1367 (2019).

24. Summers, N. A. et al. Metabolic changes associated with the use of integrase strand transfer inhibitors among virally controlled women. J Acquir Immune Defic Syndr, 85, 355-362 https://doi.org/https:10.1097/QAl.0000000000002447 (2020).

25. Cardoso, S. W. et al. Outcomes of second-line combination antiretroviral therapy for HIV-infected patients: a cohort study from Rio de Janeiro, Brazil. BMC Infect Dis, 14, 699 https://doi.org/https:10.1186/s12879-014-0699-5 (2014).

26. Napravnik, S. et al. Outcomes of second combination antiretroviral therapy regimens among HIVinfected persons in clinical care: a multicenter cohort study. AIDS Res Hum Retroviruses, 29, 574-580 https://doi.org/https:10.1089/AID.2012.0210 (2013).

27. Napravinic, S. et al. Triple-class antiretroviral drug resistance: risk and predictors among HIV-1infected patients. AIDS, 21, 825-834 (2007);21: 825-34

\section{Tables}


Table 1. Characteristics of patients who switched anchor drugelass from the lirst ART regimen $(n=3,108)$

\begin{tabular}{|c|c|c|c|c|c|c|c|c|c|c|c|c|}
\hline $\begin{array}{l}\text { Characterist ic } \\
\text { Age group (vears) }\end{array}$ & \multicolumn{3}{|c|}{$\begin{array}{l}\text { Overall } \\
\mathrm{N}=3,108\end{array}$} & \multicolumn{3}{|c|}{$\begin{array}{l}\text { NNRTI } \\
N=568\end{array}$} & \multicolumn{3}{|c|}{$\begin{array}{c}\mathrm{PI} \\
\mathrm{N}=2,057\end{array}$} & \multicolumn{3}{|c|}{$\begin{array}{l}\text { INSTI } \\
N=483\end{array}$} \\
\hline$<20$ & & - & - & & & - & & & - & & - & \\
\hline $20-29$ & & 153 & $(4.9 \%)$ & & 25 & $(4.48)$ & & 89 & $(4.3 \%)$ & & 39 & $(8.1 \%)$ \\
\hline $30-39$ & & 836 & $(25.9 \%)$ & & 145 & $(25.7 \%)$ & & 574 & $(27.9 \%)$ & & 116 & $(24.0 \%)$ \\
\hline $40-49$ & & 1,142 & $(35.7 \%)$ & & 204 & $(35.9 \%)$ & & 773 & $(37.6 \%)$ & & 165 & $(34.2 \%)$ \\
\hline $50-59$ & & 603 & $(19.4 \%)$ & & 116 & $(20.4 \%)$ & & 398 & $(19.3 \%)$ & & 89 & $(18.4 \%)$ \\
\hline $60-69$ & & 264 & $(8.5 \%)$ & & 57 & $(10.095)$ & & 160 & $(7.8 \%)$ & & 47 & $(9.7 \%)$ \\
\hline$\approx 70$ & about & 100 & - & about & 10 & - & about & 60 & - & Lout & 20 & \\
\hline \multicolumn{13}{|l|}{$\operatorname{sex}$} \\
\hline Male & & 2855 & $(91.9 \%)$ & & 529 & $(93.1 \%)$ & & 1,883 & $(91.5 \%)$ & & 443 & $(91.7 \%)$ \\
\hline Fernale & & 253 & $(8.1 \%)$ & & 39 & $(6.9 \%)$ & & 174 & $(8.5 \%)$ & & 40 & $(8.3 \%)$ \\
\hline AIDS-delining illness & & 1,632 & $(52.5 \%)$ & & 292 & $(51.4 \%)$ & & 1,048 & $(50.9 \%)$ & & 292 & $(60.5 \%)$ \\
\hline Disbetes & & 739 & $(23.8 \%)$ & & 120 & $(21.1 \%)$ & & 490 & $(23.8 \%)$ & & 129 & $(26.7 \%)$ \\
\hline Drslipidemia & & 577 & (18.6\%) & & 87 & $(15.3 \%)$ & & 398 & $(19.3 \%)$ & & 92 & $(19.0 \%)$ \\
\hline Hroertension & & 328 & $(10.6 \%)$ & & 65 & (11.4\%) & & 185 & $(9.0 \%)$ & & 78 & $(16.1 \%)$ \\
\hline Bone disorder & & 78 & $(2.5 \%)$ & & - & - & & 52 & $(2.5 \%)$ & bout & 10 & - \\
\hline Vasculer diseases & & 138 & $(4.4 \%)$ & & 25 & $(4.4 \%)$ & & 73 & $(3.5 \%)$ & & 40 & $(8.3 \%)$ \\
\hline Angina & & 74 & $(2.4 \%)$ & & 15 & $(2.6 \%)$ & & 36 & $(1.8 \%)$ & & 23 & $(4.8 \%)$ \\
\hline Stroke & & 62 & $(2.0 \%)$ & & & - & & 39 & $(1.9 \%)$ & Doul & 10 & \\
\hline Mrocardial in/raction & & - & - & & & - & & - & - & & - & \\
\hline Kidney disease & & 133 & $(4.3 \%)$ & & 22 & $(3.9 \%)$ & & 75 & $(3.6 \%)$ & & 36 & $(7.5 \%)$ \\
\hline Urolithiasis & & 86 & $(2.8 \%)$ & & 16 & $(2.8 \%)$ & & 46 & $(22 \%)$ & & 24 & $(5.0 \%)$ \\
\hline Chronic kidney disease & & 49 & $(1.6 \%)$ & & & - & & 29 & $(1,4 \%)$ & biont & 10 & \\
\hline Cancers & & 268 & $(8.6 \%)$ & & 39 & $(6.9 \%)$ & & 150 & $(7.3 \%)$ & & 79 & $(16.4 \%)$ \\
\hline AIDS-delining cancers & & 188 & $(6.0 \%)$ & & 27 & $(4.8 \%)$ & & 102 & $(5.0 \%)$ & & 59 & $(12.2 \%)$ \\
\hline Non-AIDS-delining cancers & & 103 & $(3.3 \%)$ & & 17 & $(3.0 \%)$ & & 57 & $(28 \%)$ & & 29 & $(6.0 \%)$ \\
\hline Psychialric disor ders & & 553 & $(17.8 \%)$ & & 83 & $(14.6 \%)$ & & 338 & $(16.4 \%)$ & & 132 & $(27.3 \%)$ \\
\hline Mania and Depression & & 356 & $(11.5 \%)$ & & 47 & $(8.3 \%)$ & & 219 & $(10.6 \%)$ & & 90 & $(18.6 \%)$ \\
\hline Anxious & & 260 & $(8.4 \%)$ & & 42 & $(7.4 \%)$ & & 155 & $(7.5 \%)$ & & 63 & $(13.0 \%)$ \\
\hline Psychosis & & 113 & $(3.6 \%)$ & & 13 & $(2.3 \%)$ & & 71 & $(3.5 \%)$ & & 29 & $(6.0 \%)$ \\
\hline Insomnig & & 15 & $(0.5 \%)$ & & & - & & & - & & - & \\
\hline Dementa & & - & - & & & - & & - & - & & - & \\
\hline Hepatitis B inlection & & 343 & $(11.0 \%)$ & & 60 & $(10.6 \%)$ & & 231 & $(11.2 \%)$ & & 52 & $(10.8 \%)$ \\
\hline Hepatitis C inlection & & 221 & $(7.1 \%)$ & & 36 & $(6.3 \%)$ & & 141 & $(6.9 \%)$ & & 44 & $(9.1 \%)$ \\
\hline Syphilis & & 939 & $(30.2 \%)$ & & 150 & $(26.4 \%)$ & & 635 & $(30.9 \%)$ & & 154 & $(31.9 \%)$ \\
\hline \multicolumn{13}{|l|}{ Hospilalize } \\
\hline Hospilalized & & 1436 & $(45.2 \%)$ & & 220 & $(38.7 \%)$ & & 924 & $(44.9 \%)$ & & 292 & $(60.5 \%)$ \\
\hline Never & & 1672 & $(53.8 \%)$ & & 348 & $(61.3 \%)$ & & 1.133 & $(55.1 \%)$ & & 191 & $(39.5 \%)$ \\
\hline \multicolumn{13}{|l|}{ Year ol ART initiation } \\
\hline 2011 & & 844 & $(27.2 \%)$ & & 195 & $(34.5 \%)$ & & 564 & $(27.4 \%)$ & & 84 & $(17.4 \%)$ \\
\hline 2012 & & 837 & $(25.9 \%)$ & & 137 & (24.1\%) & & 637 & $(310 \%)$ & & 63 & $(13.0 \%)$ \\
\hline 2013 & & 671 & $(21.6 \%)$ & & 120 & (21.1\%) & & 488 & $(23.7 \%)$ & & 63 & $(13.0 \%)$ \\
\hline 2014 & & 351 & (11.3\%) & & 64 & (11.3\%) & & 202 & $(9.8 \%)$ & & 85 & $(17.6 \%)$ \\
\hline 2015 & & 167 & $(5.4 \%)$ & & 22 & $(3.9 \%)$ & & 75 & $(3.6 \%)$ & & 70 & $(14.5 \%)$ \\
\hline 2016 & & 110 & $(3.5 \%)$ & & 15 & $(2.6 \%)$ & & 43 & $(21 \%)$ & & 52 & $(10.8 \%)$ \\
\hline 2017 & & 81 & $(2.6 \%)$ & & - & - & |l & 20 & - & & 43 & $(8.9 \%)$ \\
\hline 2018 & abouk & 40 & - & & - & - & th & 10 & - & Lowl & 20 & - \\
\hline 2019 & & - & - & & - & - & & - & - & & - & - \\
\hline
\end{tabular}

Excluded "non-swilcher" Irom Supplement Table 2)

ART: antir etroviral therapy, NNTI: non-ncleoside reverse transcriptase inhibitor, PI: protease irhibitor, INSTI: integrase strand transer inhibitor Vahyes are expressed as nuber (percentap) unless specified otherwise 


\begin{tabular}{|c|c|}
\hline $\begin{array}{r}\text { Disease name } \\
\end{array}$ & ICD-10 code \\
\hline \multicolumn{2}{|l|}{ ADS-d fining illnesses } \\
\hline HIV non-tuberculous nycobacteri a & B20.0 \\
\hline HIV cytomegalovirus infection & B20.2 \\
\hline HIV other viral infections & B20.3 \\
\hline HIV candidiasis & B20.4 \\
\hline HIV Pnevmocystis carinii pnevmonia & B20.6 \\
\hline HIV Kaposi's sarcoma & B21.0 \\
\hline HIV Burkitt's lymphoma & B21.1 \\
\hline HIV non-Hodglin's lymphoma & B21.2 \\
\hline HIV encephal opathy & $\mathrm{B} 22.0$ \\
\hline HIV lymphoid interstitial pneumonitis & B22.1 \\
\hline Slim disease & B22.2 \\
\hline HIV other specified conditions & B23.8 \\
\hline AIDS & B 24 \\
\hline AIDS-related complex & B 24 \\
\hline Mal ignant neoplasm of the cervix uteri & $\mathrm{C} 53$ \\
\hline \multicolumn{2}{|l|}{ AIDS-d fining cancers } \\
\hline Kaposi sarcoma & $\mathrm{B} 21.0, \mathrm{C} 46$ \\
\hline Burkitt lymphoma & B 21.1, C 83.7 \\
\hline Non-Hodgkin lymphoma & $\mathrm{B} 21.2, \mathrm{C} 82-\mathrm{C} 85$ \\
\hline Mal ignant neoplasm of the cervix uteri & $\mathrm{C} 53$ \\
\hline \multicolumn{2}{|l|}{ Non-AIDS-defining cancers } \\
\hline Cancer other than the above & C00-97 Exclude C46, C53, C82-C85, C83.7) \\
\hline \multicolumn{2}{|l|}{ Comorbidities } \\
\hline HIV related diseases & \\
\hline Cytomegaloviral disense & $\mathrm{B} 25, \mathrm{~B} 45, \mathrm{~B} 59, \mathrm{C} 46.9, \mathrm{C} 81$ \\
\hline Hypertension & $\mathrm{I} 10, \mathrm{I} 12, \mathrm{I} 14-15$ \\
\hline Dyslipidemia & $\cdot$ \\
\hline Hypercholesterolaenia or hyperlipidaemia & E78.0-78.5 \\
\hline Hepatitis B infection & B18.1 \\
\hline Hepatitis C infection & B18.2 \\
\hline Diabetes & \\
\hline Type 2 diabetes & E11-14 \\
\hline Bone disorder & \\
\hline Osteoporosis & M80-81 \\
\hline Vascular disease & $\cdot$ \\
\hline Myocardial infarction & I $21-22$ \\
\hline Stroke & I64 and related receipt diagnosis codes \\
\hline Angina pectoris & I 20 \\
\hline Hypertensive heart and renal diseases & $\mathrm{I11}, \mathrm{I13}$ \\
\hline Psychiatric disorders & \\
\hline Mania and depression & F $30-32$ \\
\hline Anxiety & F $40-41$ \\
\hline Poychosis & F $20-29$ \\
\hline Dementia & $\mathrm{F} 01, \mathrm{~F} 03$ \\
\hline Insomnia & F51 \\
\hline Kidney disease & - \\
\hline Chronic lidney disease & N18-19 \\
\hline Urolithiasis & $\mathrm{N} 20-21$ \\
\hline Malignancies & B21.0-21.2, C $00-97$ \\
\hline Syphilis & $\mathrm{A} .53$ \\
\hline
\end{tabular}

\section{Figures}


first anchor drug classes by year

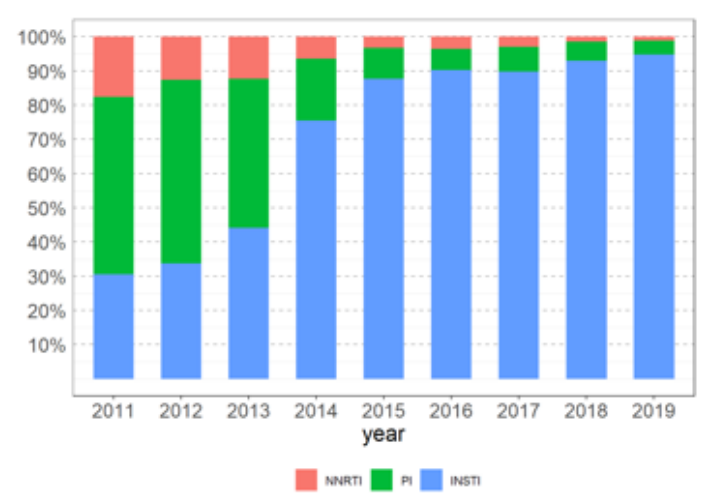

first backbone drugs by year

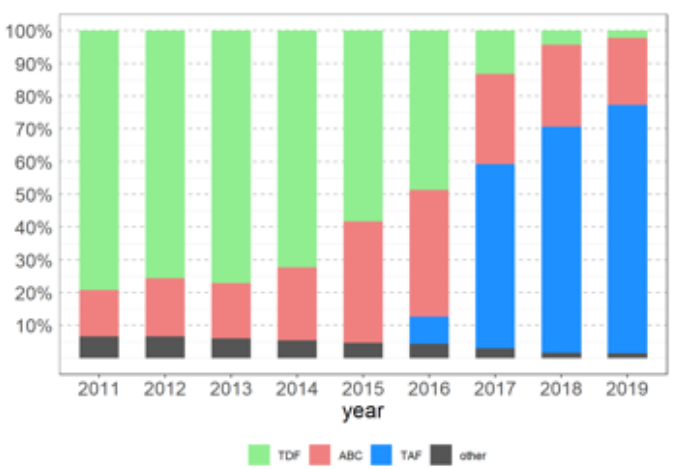

\begin{tabular}{|c|c|c|c|c|c|c|c|c|c|c|c|c|c|c|c|c|}
\hline \multirow{4}{*}{$\begin{array}{l}\text { Timing of switch } \\
(\mathrm{n}=3108)\end{array}$} & \multicolumn{16}{|c|}{ Anchor drug class in the second regimen } \\
\hline & \multicolumn{7}{|c|}{ Switch $<1$ year after starting the first regimen } & \multicolumn{9}{|c|}{ Switch $\geqq 1$ year after starting the first regimen } \\
\hline & \multicolumn{2}{|l|}{ NNRTI } & \multicolumn{3}{|l|}{ PI } & \multicolumn{2}{|l|}{ INSTI } & \multicolumn{3}{|c|}{ NNRTI } & \multicolumn{3}{|c|}{$\mathrm{PI}$} & \multicolumn{3}{|l|}{ INSTI } \\
\hline & n $\%$ & $(95 \% \mathrm{Cl})$ & $\mathrm{n}$ & $\%$ & $(95 \% \mathrm{Cl})$ & $\mathrm{n} \quad \%$ & $(95 \% \mathrm{Cl})$ & $n$ & $\%$ & $(95 \% \mathrm{Cl})$ & $\mathrm{n}$ & $\%$ & $(95 \% \mathrm{Cl})$ & $n$ & $\%$ & $(95 \% \mathrm{Cl})$ \\
\hline NNRTI & $=$ & $\cdot$ & 56 & $(32.7 \%)$ & $(29.6-36.1)$ & $115(67.3 \%)$ & $(63.9-70.4)$ & $\cdot$ & - & $=$ & 17 & $(4.3 \%)$ & $(3.4-5.3)$ & 379 & $(95.5 \%)$ & $(94.4-96.3)$ \\
\hline $\mathrm{PI}$ & $61(12.5 \%)$ & $(11.2-13.9)$ & - & - & $\cdot$ & $420(85.9 \%)$ & $(84.4-87.3)$ & 87 & $(5.5 \%)$ & $(5.0-6.1)$ & - & - & $=$ & 1467 & $(93.6 \%)$ & $(93.0-94.1)$ \\
\hline INSTI & $88(28.6 \%)$ & $(26.3-31.0)$ & 202 & $(65.6 \%)$ & $(63.1-68.0)$ & - & - & 62 & $(35.4 \%)$ & $(32.2-38.8)$ & 97 & $(55.4 \%)$ & $(52.0-58.8)$ & - & - & - \\
\hline
\end{tabular}

\section{Figure 1}

Distribution of (a) anchor and (b) backbones drug classes prescribed in first ART regimen by year (20112019) $(n=16,069)$ 


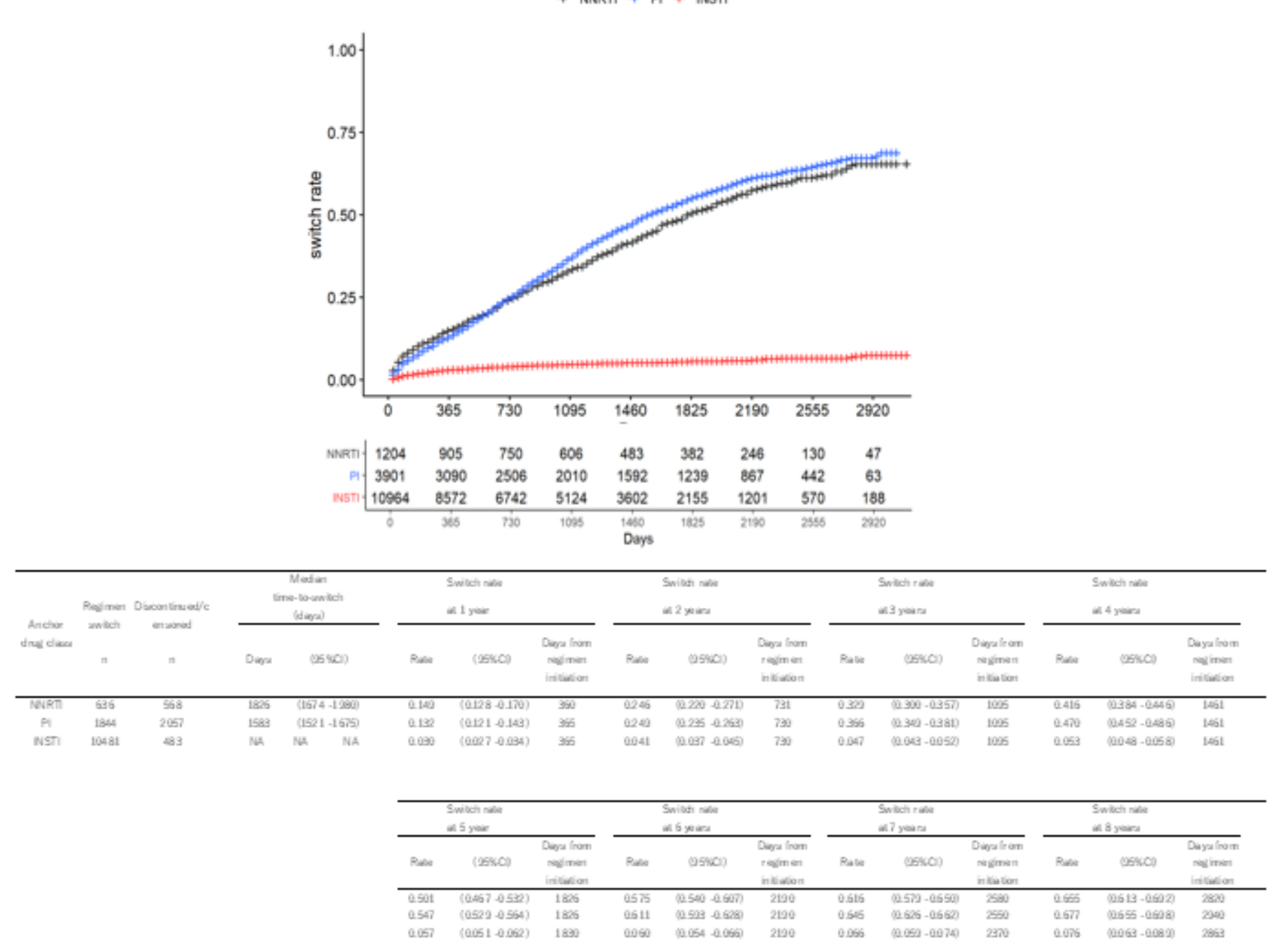

\section{Figure 2}

Time-to-switch of ART regimens according to anchor drug class from 2011-2019 


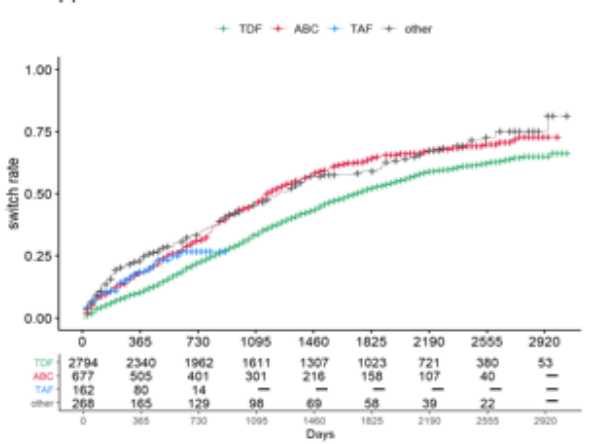

NNRTI

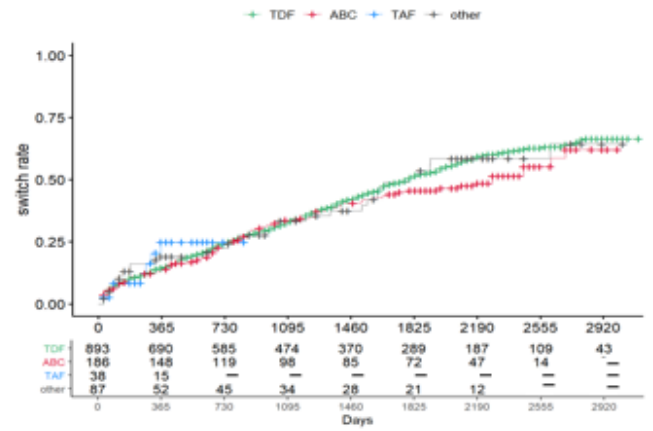

INSTI

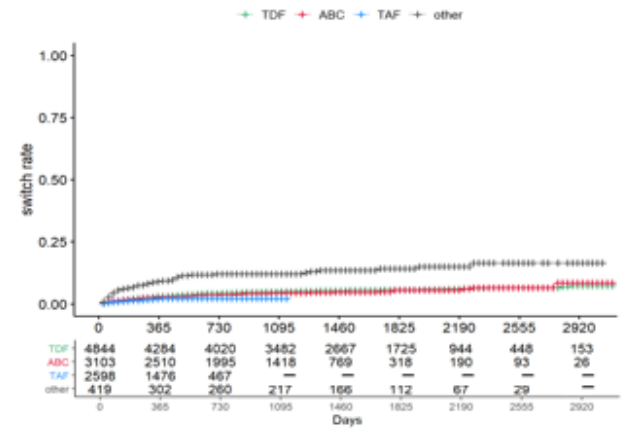

\begin{tabular}{|c|c|c|c|c|c|c|c|c|c|c|}
\hline \multirow{3}{*}{$\begin{array}{l}\text { Ancior } \\
\text { drug dien }\end{array}$} & \multirow{3}{*}{ Busctione } & \multicolumn{2}{|c|}{ 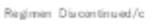 } & \multirow{2}{*}{\multicolumn{2}{|c|}{ 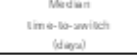 }} & \multirow{2}{*}{\multicolumn{2}{|c|}{$\begin{array}{l}\text { Snibeh rate } \\
41 \text { yese }\end{array}$}} & \multirow{3}{*}{ Provos } & & \multirow{3}{*}{ 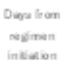 } \\
\hline & & moteh & arooned & & & & & & & \\
\hline & & & & $D_{\text {owy }}$ & & Rasta & (askon & & & \\
\hline \multicolumn{11}{|l|}{ NWPT } \\
\hline & TOF & $\Leftrightarrow$ & 45 & 1725 & $(1600-1072)$ & 0.45 & $0.121-0.152)$ & TDF $v y A B C$ & 1 & 350 \\
\hline & $\triangle B C$ & 106 & 82 & 2280 & (158A -NA) & 0.140 & (20.87-0.189) & TOF $v a$ TAF & 1 & she \\
\hline & TAE & 31 & & NA & NA NA & Q2.249 & Q.66 - -2937$)$ & TOF vat oben & 1 & 90 \\
\hline & seters & 53 & 34 & 1726 & $(1529$-NA) & 2019 & $0.095-0.277)$ & $A B C$ vy TAF & 1 & 995 \\
\hline & & & & & & & & $A B C$ wa dien & 1 & \\
\hline & & & & & & & & DAFva othen & 1 & \\
\hline \multicolumn{11}{|c|}{ moos 01489} \\
\hline & TOF & 1305 & 14 & 1740 & $(1630-1827)$ & Q 107 & $0.05-0.118)$ & TDF $v \backsim A B C$ & $<0.05$ & 365 \\
\hline & 250 & 2at & 326 & 1170 & $(1005-1302)$ & 2.186 & $0.156-0.216)$ & TOF $v a$ TAE & 0.082 & 355 \\
\hline & TAF & (19) & 32 & NA & NA NA & Q.187 & $(0.118-0.251)$ & TDF vat other & $<005$ & 30 \\
\hline & othera & 127 & 141 & 1230 & $(1000-1520)$ & 2235 & $0+2-0.287)$ & $A B C$ vy TAF & 1 & 355 \\
\hline & & & & & & & & $A B C$ va when & 1 & \\
\hline & & & & & & & & TAFvy othes & 1 & \\
\hline \multirow{2}{*}{\multicolumn{11}{|c|}{$0027-0.0977$}} \\
\hline & TOF & 565 & 252 & NA & NA NA & 0032 & $0.027-0.097)$ & TOF $v x A B C$ & 1 & 965 \\
\hline & $A B C$ & $2 n$ & 126 & NA & NA NA & 2028 & $(0.22-0.034)$ & TOF va TAF & 0002 & 366 \\
\hline & $T A F$ & 250 & 48 & NA & NA NA & 0201 & $0.915-0027)$ & TOF vat obley & 2005 & 965 \\
\hline & onera & sa & so & NA & NA NA & 2004 & $0.064-0.123)$ & ABC veTAF & 0004 & 956 \\
\hline & & & & & & & & $A B C$ wa seley & $<005$ & \\
\hline & & & & & & & & TAFvy othes & $<0.05$ & \\
\hline
\end{tabular}

\section{Figure 3}

Time-to-switch of ART regimens by anchor drug class-based backbone type from 2011-2019 
AIDS or not

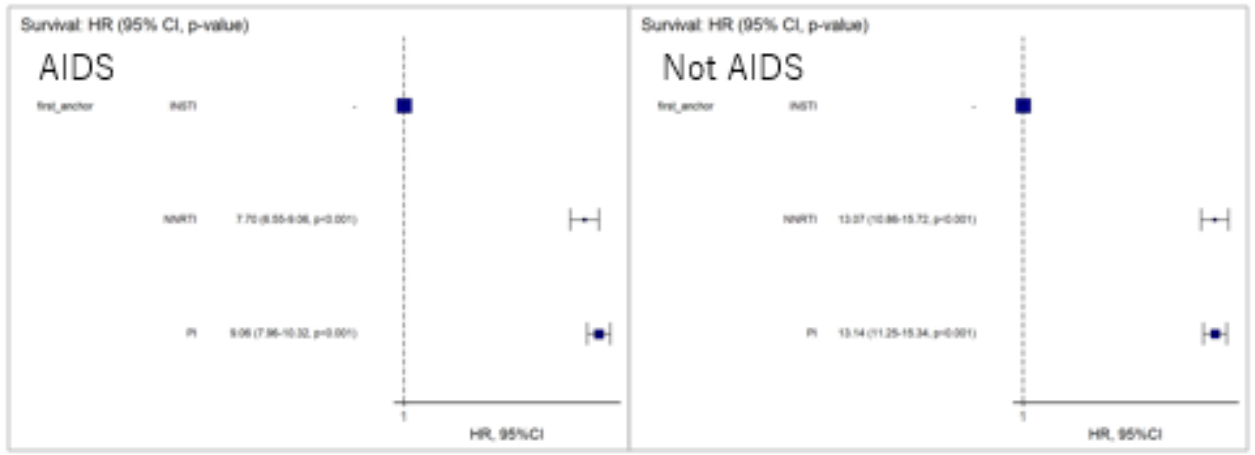

\section{Backbone}

Sunvat HR (95\% Ci, p-vatue)

$\mathrm{ABC}$

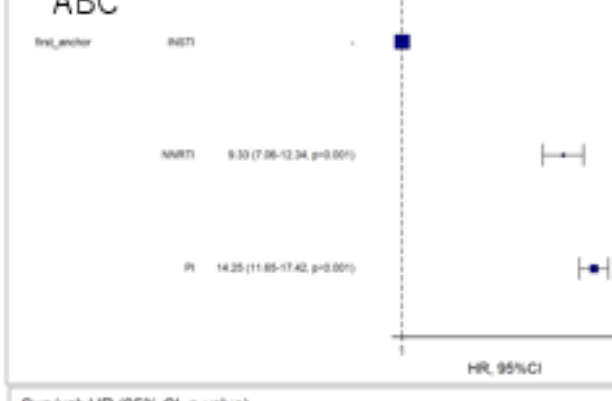

Survivat HR (95\% Cl, p-vatue)

TAF

mon

man

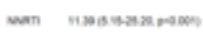

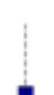

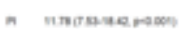

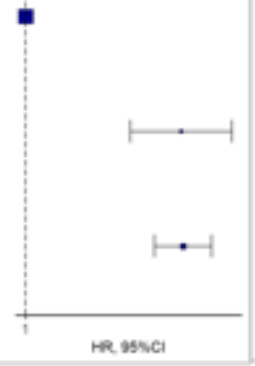

Sunviat HR (95: $\mathrm{Cl}$. pintu) TDF m.n. nam

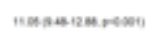

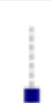

a smasoras poser
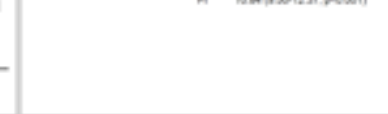

Sunviat HR (95\% Cl, p-vatue)

Ha. ostat

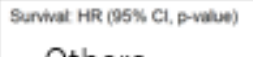
Others man

mati indesum mose

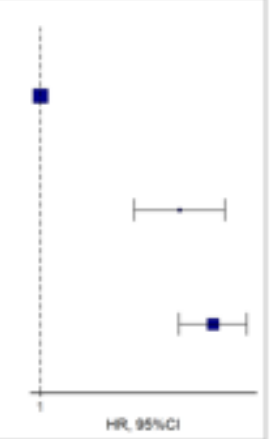

\section{Figure 4}

Hazard ratio for switching of each anchor drug class stratified by AIDS-defining illness and backbone drug.

\section{Supplementary Files}

This is a list of supplementary files associated with this preprint. Click to download.

- Onlinefloatimage2.png

- Onlinefloatimage3.png

- SuppFig1.png 